\title{
Biología reproductiva de cinco especies ornitófilas en un fragmento de bosque caducifolio secundario en Venezuela
}

\author{
C. Grases y N. Ramírez
}

Universidad Central de Venezuela. Instituto de Biología Experimental. Centro de Botánica Tropical. Laboratorio de Biología Reproductiva. Apartado 20513. Caracas, Venezuela.

Recibido 1-X-1997. Corregido 7-VIII-1998. Aceptado 20-VIII-1998.

\begin{abstract}
The floral biology, the pollination biology and the mating system were studied in two native and three introduced species of an arboretum in Venezuela. All the species presented thin, long, tubular corollas; predominantly red. The antheses were diurnal (between 04:00 and 11:00 hr). Nectar production was high, except for Salvia coccinea (Lamiaceae) (native). Nicotiana glauca (Solanaceae) (native) and Erythrina mitis (Fabaceae) (introduced) shared their hummingbird pollinators, Chlorostilbon mellisugus and Amazilia tobaci (Apodiformes, Throchilidae). S. coccinea was pollinated by Heliconius erato (Lepidoptera). Kalanchöe pinnata and $K$. daigremontiana (Crassulaceae) (exotic) did not receive any visits. All the species were hermaphrodite. $K$. daigremontiana and $K$. pinnata were obligate outcrossers, and $S$. coccinea, $N$. glauca and $E$. mitis were partialy outcrossers and non-autogamus. The species presented high total biomass allocation, compared to other polination systems. The reproductive biomass allocation were allways smaller than the attraction and vegetative biomass allocation. The male biomass allocation were greater than the female biomass allocation in $N$. glauca, $S$. coccinea and $E$. mitis; and smaller in $K$. daigremontiana and $K$. pinnata. The introduced species did not seem to affect the reproductive ef ficiencies of the native species during our observation period. The lowest reproductive efficiencies in introduced species resulted as a consequence of the absence of their natural pollinators.
\end{abstract}

Key words: Hummingbirds, hummingbird pollination, deciduous forest, biomass allocation, reproductive system, reproductive eficiency, introduced species.

En las comunidades naturales neotropicales coexisten plantas ornitófilas y especies de colibríes. Las especies vegetales podrían compartir sus polinizadores (Stiles 1978, 1979, Neill 1987, Feinsinger \& Tiebout III 1991, Feinsinger et al. 1986, 1991), generando competencia y afectando así el rendimiento de sus procesos individuales de polinización (Feinsinger et al. 1991). Dicha competencia es reducida al disminuir el solapamiento fenológico (Stiles 1978, 1979, Feinsinger \& Tiebout III 1991); y segregando la ubicación de las cargas de polen sobre el polinizador (Feinsinger \& Tiebout III 1991, Ramírez 1992). Todo lo anterior resulta de estudios realizados en comunidades o gremios naturales, cuya composición florística, en su gran mayoría, es de especies nativas. En la actualidad existen fuertes presiones antrópicas sobre las áreas naturales de vegetación, encontrándose frecuentemente áreas fuertemente intervenidas. Esta situación es mucho más crítica en áreas urbanas, donde solo fragmentos de vegetación permanecen en forma aislada. Bajo este marco actual, las 
pautas de reproducción, a nivel comunitario, pueden estar severamente afectadas (Feinsinger et al. 1987, Linhart et al. 1987). Sin embargo, el efecto de la perturbación de la vegetación sobre los procesos reproductivos es poco conocida, así como el tipo de interacciones que se presentan cuando se introducen plantas exóticas y no exóticas a una comunidad secundaria. La presencia de especies introducidas pudieran afectar negativamente los procesos de polinización de las especies nativas, en el caso que los polinizadores fueran comunes.

De acuerdo a lo anterior se plantean las siguientes preguntas: ¿Cuáles serían las eficiencias individuales de las especies nativas e introducidas, y cuales serían las interacciones planta-planta y planta-polinizador en una comunidad en cuya composición florística coexistan especies nativas e introducidas? Si estas interacciones requieren de una esçala temporal evolutiva para lograr un equilíbrio, entonces la presencia de especies introducidas podría tener algún efecto sobre los procesos reproductivos. Por otro lado, si las especies introducidas son polinizadas por vectores específicos, en sus áreas de distribución natural, entonces la eficiencia de los visitantes florales diferentes podría ser distinta, variando la eficiencia total del sistema.

Este trabajo caracteriza la biología floral, biología de polinización y sistema de apareamiento de cinco especies ornitófilas de un fragmento de bosque caducifolio secundario en Venezuela, con la intención de conocer sus características individuales y sus interacciones planta-planta y planta-polinizador, así como evaluar comparativamente la eficiencia reproductiva entre especies naturales y exóticas, bajo condiciones de alteración secundaria de la vegetación.

\section{MATERIALES Y MÉTODOS}

Area de estudio: El presente trabajo fue realizado en el Arboretum del Instituto de Biología Experimental de la Universidad Central de Venezuela; Colinas de Bello Monte, Estado Miranda, Venezuela ( $\left.10^{\circ} 30^{\prime} \mathrm{N}, 66^{\circ} 53^{\prime} \mathrm{W}\right)$, a una altitud de $1.100 \mathrm{~m}$ s.n.m. Esta reserva corresponde a un bosque seco premontano (Ewel et al. 1976), con una precipitación media anual que varía entre 550 y $1.100 \mathrm{~mm} 3$; y una temperatura que varía entre $18^{\circ}$ y $24^{\circ} \mathrm{C}$. Esta reserva contiene 2 hectáreas de vegetación caducifolia secundaria continua, considerada sucesional (Ewel et al. 1976), consistiendo en dos estratos pobremente definidos: 1- un estrato arbóreo de 5-8 $\mathrm{m}$ de altura, carente de especies dominantes; y 2- un estrato más bajo, que contiene arbustos, hierbas y especies suculentas; estas últimas probablemente representando la vegetación colonizadora original (Ruiz \& Arroyo 1978). Algunas de las plantas introducidas fueron sembradas, mientras que otras germinaron de forma espontánea. Tamayo (1943) informa la primera alteración de la vegetación y erosión del suelo entre los años 1936-37. Se han sucedido numerosas alteraciones hasta los momentos actuales. Las características de las especies estudiadas se encuentran reseñadas en el Cuadro 1.

\section{CUADRO 1}

Hábito, porte, condición y período de floración de cinco especies ornitófilas de un fragmento de bosque caducifolio secundario en Venezuela

\begin{tabular}{|c|c|c|c|c|c|}
\hline Especies & Familia & Hábito & Porte & Condición & Período de floración \\
\hline Salvia coccinea. Juss. ex Murr. & Lamiaceae & Herbáceo & Hasta $1 \mathrm{mt}$ & Nativa & Abr -Dic \\
\hline icotiana glauca. Grah. & Solanaceae & Arbóreo & $3-6 \mathrm{mts}$ & Nativa & Dic -Todo el año \\
\hline Erythrina mitis. Jacq. & Fabaceae & Arbóreo & $4-9 \mathrm{mts}$ & Introducida & $\mathrm{Dic}-\mathrm{Abr}$ \\
\hline $\begin{array}{l}\text { Kalanchöe pinnata. (Lam.) Pers. } \\
\text { Kalanchöe daigremontiana. }\end{array}$ & Crassulaceae & Herbáceo & Hasta $1,5 \mathrm{mts}$ & Exótica & Dic - Feb \\
\hline R. Hamet \& H. Perr. de la Bath. & Crassulaceae & Herbáceo & Hasta $2 \mathrm{mts}$ & Exótica & Oct - Feb \\
\hline
\end{tabular}


Biologia floral: El análisis morfológico de las flores se basó en los tipos florales propuestos por Faegri y van der Pijl (1979). Asimismo, el color floral se determinó por comparación con el atlas de colores de Küppers (1979). Las dimensiones florales (largo y diámetro del cáliz, de la corola, del androceo y del gineceo) se estimaron para un total de 20 flores con material fresco. También se determinó si las estructuras reproductivas (androceo y gineceo) se encuentran insertas o exertas a la corola. La orientación de la flor se determinó definiendo tres criterios: vertical hacia arriba, cuando la flor se orienta en el sentido de la fuerza de la gravedad y la abertura de la corola se encuentra opuesta a la superficie del suelo; vertical hacia abajo, cuando la flor se orienta en el sentido de la fuerza de la gravedad y la abertura de la corola se encuentra orientada hacia la superficie del suelo; y horizontal, cuando la flor se orienta de forma perpendicular en sentido de la fuerza de la gravedad.

El proceso de antesis fue estimado en flores de diferentes individuos, cuantificando el número de flores marcadas que abren en intervalos de media hora o de una hora, en un mínimo de 20 flores. La longevidad fue estimada por observaciones periódicas cada $24 \mathrm{hr}$, exceptuando el caso de Salvia coccinea en donde las observaciones fueron realizadas cada hora; desde la antesis hasta la marchitez de la flor.

El tamaño del grano de polen se determinó en 10 granos, utilizando un microscopio con micrómetro ocular. Posteriormente, el área del grano de polen se estimó utilizando la media del radio de los granos (debido a que no todo el área del grano de polen entra en contacto con el área estigmática, sólo se tomó la mitad del área del grano de polen para obtener un resultado mas aproximado del área ocupada por el grano de polen). Por otra parte, el área estigmática se estimó en 10 estigmas utilizando una cámara clara. La cámara clara permite dibujar el área plana estigmática sobre un papel (realizando el número de cortes que sea necesario para que el área estigmática se encuentre bidimensional), para luego cortar y pesar las áreas dibujadas. Este peso se convirtió a un área luego de conocer el peso de $1 \mathrm{~cm}^{2}$ del papel. Por último, se estimó la relación área estigma/área polen dividiendo los resultados anteriores. La relación área estigma/área polen es un parámetro que indica el número de granos de polen que potencialmente puede ocupar la totalidad del área estigmática receptiva en una sola capa.

Para estimar la producción de néctar, se realizaron medidas con microcapilares en 20 flores aisladas recién abiertas. En flores muy angostas, la corola fue cortada, realizándose una sola medida por flor y obteniendo un valor de néctar acumulado. La columna de néctar en el capilar fue medida y se transformó a volumen de acuerdo al diámetro interno del tubo capilar.

Biología de polinización: Las especies polinizadoras y modo de polinización fueron estimados por observación directa de la actividad de los agentes visitantes, relacionado con parámetros como recompensa, antesis y longevidad. La abundancia y posición de las cargas de polen fueron determinadas cualitativamente por observación directa, utilizando un microscopio estereoscópico, en el caso de los insectos visitantes. Para el caso de las aves fue determinada por observaciones de campo utilizando binóculos, debido a que no se pudo capturar ningún ejemplar. La relación entre el tamaño flor-polinizador, posición de las cargas de polen, y el comportamiento durante la visita fueron los criterios principales para diferenciar visitantes de polinizadores. La identificación de los ejemplares de colibríes fue realizada por comparación directa con las descripciones de Phelps y de Schauensee (1979).

Sistema de apareamiento: La caracterización de la sexualidad floral se basó en observaciones directas en el campo, determinándose de acuerdo a la presencia de estambres funcionales y la presencia de un ovario. La separación espacial de estambres y estigmas, hercogamia (Webb \& Lloyd 1986), es definida utilizando el criterio de Ramírez (1993), quién toma una distancia mínima de $2 \mathrm{~mm}$ de separación del estigma sobre las anteras. Las flores 
fueron separadas en flores adicógamas, cuando la dehiscencia de las anteras y la receptividad del estigma ocurren simultáneamente; y en dicógamas, cuando la receptividad del estigma y la dehiscencia de las anteras no ocurren simultáneamente (LLoyd \& Webb 1986).

El sistema de apareamiento se estimó realizando 4 pruebas controladas siguendo la metodología descrita por Ruiz y Arroyo (1978) y Sobrevila y Arroyo (1982). La prueba de autofertilidad se realizó en flores recién abiertas, aisladas previamente por medio de bolsas de material sintético, y autofertilizadas manualmente. La prueba de fertilización cruzada se realizó colocando polen proveniente de diferentes individuos en estigmas receptivos de flores emasculadas y previamente aisladas para evitar la polinización accidental. Los niveles de autogamia se determinaron en yemas aisladas bien desarrolladas, no manipuladas hasta la formación de frutos. Finalmente, la agamospermia se estimó emasculando yemas florales y aislándolas. Para cada tipo de prueba aplicada se cuantificó el número de frutos y semillas normales producidas. Plantas que produjeron valores de frutos y semillas por autofertilización significativamente menores a los producidos por fertilización cruzada, fueron considerados autoincompatibles. De igual manera, plantas que produjeran valores de frutos y semillas por polinización automática significativamente menores a los producidos por autofertilización, fueron considerados como no-autógamas. El estadístico empleado para las comparaciones fue la prueba de G. a $\mathrm{P}<0.001$, modificado de Ramírez \& Seres (1994).

El número de granos de polen por flor se estimó de forma indirecta según la metodología descrita por Lloyd (1965), el cual consiste en suspender 5 anteras de diferentes yemas florales en una solución de anilina en lactofenol y glicerina en proporciones de 3:1. Posteriormente se cuantificaron los granos de polen por triplicado en un volumen conocido, con un hematocitómetro y directamente al microscopio. Para todas las variables anteriores se procedió a determinar las siguientes variables estimadas: porcentaje de semillas abortadas; porcentaje de óvulos abortados; porcentaje de flores-frutos abortados y la relación polen/óvulo (P/O).

Biomasa: Se estimó el peso seco de las partes florales; cáliz, corola, anteras, filamentos y gineceo. Esto se hizo en 20 flores en antesis, secadas en una estufa a $40^{\circ} \mathrm{C}$ hasta peso constante. Luego se pesaron en una balanza analítica. El peso de las anteras fue obtenido utilizando yemas muy cercanas a su antesis para asegurar que las anteras se encontraran totalmente desarrolladas y cerradas. Posterior a esto, se estimaron las relaciones biomasa androceo/biomasa gineceo (BAnd/BGin); biomasa reproductiva/ biomasa de atracción (BR/BA); y biomasa reproductiva/ biomasa vegetativa (BR/BV). Por biomasa de atracción se entiende aquella asociada a la corola, por biomasa vegetativa se entiende la suma de la biomasa asociada al cáliz y a la corola, y por biomasa reproductiva se entiende la suma de las biomasas asociadas al androceo y al gineceo.

Eficiencia natural: El número de flores por inflorescencia y de frutos por infrutescencia se determinaron por conteos directos en 100 inflorescencias e infrutescencias respectivamente. Las determinaciones del número de óvulos por ovario y de semillas por fruto se realizaron por conteos directos bajo el microscopio estereoscópico en 100 ovarios y 100 frutos respectivamente. Se cuantificó el número total de semillas por fruto, discriminándose las semillas abortivas y las normales.Se determinaron tres niveles de aborto: aborto de floresfrutos, aborto de óvulos y aborto de semillas.

\section{RESULTADOS}

Biología floral: Todas las especies estudiadas presentaron la inflorescencia como unidad de atracción, a excepción de Salvia cocci$n e a$, en cuyo caso, la unidad de atracción fue la flor. Por otro lado, las cinco especies estudiadas presentaron a la flor como unidad de polinización y un predominio de los colores rojos a nivel floral (Cuadro 2). Todas las especies 


\section{CUADRO 2}

Características florales de cinco especies ornitófilas de un fragmento de bosque caducifolio secundario en Venezuela

\begin{tabular}{|c|c|c|c|c|c|c|c|c|c|c|}
\hline \multirow[b]{2}{*}{ Especies } & \multicolumn{10}{|c|}{ Dimensiones Florales $(\mathrm{cm})$} \\
\hline & $\begin{array}{l}\text { Unidad de } \\
\text { olinización }\end{array}$ & Simetría & Color & Largo Corola & $\begin{array}{c}\text { Diámetro } \\
\text { Mínimo Interno }\end{array}$ & $\begin{array}{c}\text { Distancia } \\
\text { Antera-Estigma }\end{array}$ & $\begin{array}{l}\text { Exce } \\
\text { Anter }\end{array}$ & $\begin{array}{l}\text { ón } \\
\text { stigma }\end{array}$ & $\begin{array}{c}\text { Antesis I } \\
\text { (horas) }\end{array}$ & $\begin{array}{l}\text { Longevidad } \\
\text { (horas) }\end{array}$ \\
\hline Nicotiana glauca & flor & Ac & Am & $3.30 \pm 0.152$ & $0.602 \pm 0.032$ & $0.1204 \pm 0.416$ & no & no & $6: 30-7: 30 \mathrm{am}$ & 48 \\
\hline Salvia coccinea & flor & $\mathrm{Zg}$ & $\mathbf{R j}$ & $2.321 \pm 0.415$ & $0.163 \pm 0.047$ & $0.11 \pm 0.117$ & si & si & $4: 00-4: 30$ am & n $11-12$ \\
\hline $\begin{array}{l}\text { Erythrina mitis } \\
\text { Kalanchöe }\end{array}$ & flor & $\mathrm{Zg}$ & $\mathrm{Rj}-\mathrm{Fc}$ & $6.67 \pm 0.188$ & $0.413 \pm 0.012$ & 0 & no & no & $8: 00-9: 00 \mathrm{am}$ & 72 \\
\hline daigremontiana & flor & Ac & $\mathrm{Fc}$ & $3.008 \pm 0.078$ & $0.313 \pm 0.016$ & $0.258 \pm 0.106$ & no & no & $10: 00-11: 00$ an & $\operatorname{ml} 144-168$ \\
\hline Kalanchöe pinnato & ta flor & Ac & $\mathrm{Fc}-\mathrm{Vr}$ & $5.276 \pm 0.18$ & $0.321 \pm 0.10$ & $0.085 \pm 0.044$ & no & no & $6: 00-8: 00$ am & 144 \\
\hline
\end{tabular}

Ac. actinomorfa; $\mathrm{Zg}$, zigomorfa; Am, amarillo; $\mathrm{Rj}$, rojo; Fc, fucsia; Vr, verde;

presentaron corolas tubulares. S. coccinea y Erythrina mitis presentaron corolas zigomorfas; y Nicotiana glauca, Kalanchöe daigremontiana y $K$. pinnata presentaron corolas actinomorfas (Cuadro 2). Todas las especies presentaron corolas largas y estrechas (Cuadro 2). La orientación de las flores resultó ser horizontal para todas las especies, a excepción de las dos especies de Kalanchöe. S. coccinea fue la única especie cuyas estructuras reproductivas (androceo y gineceo) estaban exertas a la corola. La antesis de todas las especies fue diurna, entre las 04:00 y las 11:00, y los resultados con respecto a la longevidad floral fueron muy variables: desde $12 \mathrm{hr}$ ( $S$. coccinea), hasta 7 días (K. daigremontiana). En el Cuadro 1 se muestran los períodos de floración de cada especie estudiada. Se observa una coincidencia general de las plantas introducidas a florecer en el período seco del año. $S$. coccinea floreció en el período de lluvia y $N$. glauca fue la única especie con floración continua a lo largo del año.

Los resultados de producción de néctar están resumidos en el Cuadro 3. La producción de néctar varió entre $1.606 \mu l$ ( $S$. coccinea) y $65.5 \mu \mathrm{l}$ ( $K$. pinnata). Las especies con mayor producción total de néctar fueron $E$. mitis y $K$. pinnata. $S$. coccinea fue la única especie cuya cantidad total de néctar decreció al final de su período de longevidad floral. Algunas flores de $N$. glauca no produjeron néctar.

Biología de polinización: $N$. glauca y $E$. mitis compartieron sus polinizadores Chloros- tilbon mellisugus y Amazilia tobaci (Apodiformes, Trochilidae). El 100\% de las visitas recibidas por $N$. glauca fueron legítimas, y la deposición de polen ocurrió a lo largo de toda la superficie del pico y su base; no así para $E$. $m i$ tis, donde ambos polinizadores presentaron el siguiente comportamiento: visitas legítimas a lo largo de la abertura ventral de la flor, y visitas ilegítimas en la base de la flor. La tendencia de los patrones de las visitas de estos colibríes revelaron una intensa actividad entre las $07: 00$ y las $08: 00$ y entre las $16: 30$ y las $18: 30$. E. mitis recibió la visita ilegítima de Coereba flaveola luteola (Passeriforme, Coerebidae), la cual presentó picos de actividad entre las 09:00 y las 11:00, y las 15:00 y 16:00. Se observó un comportamiento territorialista de C. mellisugus en $E$. mitis.

S. coccinea recibió visitas legítimas de un colibrí no identificado y de Heliconius erato (Lepidoptera), siendo éste último un polinizador secundario pues presentó cantidades de polen de regular a escaso (Cuadro 4), sin embargo, es el polinizador de mayor actividad. También fue visitada por Trigona (Nanotrigona) testaecicornis (Hymenoptera, Apidae), recolectora de polen, la cual podría estar actuando como un polinizador etodinámico.

$K$. daigremontiana y $K$. pinnata no recibieron visitas legítimas. $K$. daigremontiana recibió una sola visita de Agelala multipicta (Hymenoptera) en 15 hr de observación. Para el caso de $K$. pinnata, se encontró un $100 \%$ de flores perforadas a nivel de la base de la flor 
CUADRO 3

Producción de néctar acumulado con respecto al tiempo de vida floral, de cinco especies ornitófilas de un fragmento de bosque caducifolio secundario en Venezuela

Néctar acumulado $(\mu \mathrm{l}) / \#$ Horas posteriores a la antesis

\begin{tabular}{|c|c|c|c|c|c|c|c|c|c|c|c|c|c|c|}
\hline Espécie & 0 & $1 / 2$ & $11 / 2$ & $31 / 2$ & $51 / 2$ & $71 / 2$ & $91 / 2$ & 12 & 24 & 48 & 72 & 96 & 120 & TOTAL \\
\hline N. glauca & $9.365 \pm 5.801$ & & & & & & & & $875 \pm 8.432$ & 16. $354 \pm 4.979$ & & & & $16.354 \pm 4.979$ \\
\hline S. coccinea & $0.29 \pm 0.32$ & $0.33 \pm 0.38$ & $0.89 \pm 0.18$ & $31.23 \pm 0.37$ & $1.57 \pm 0.25$ & $1.606 \pm 0.35$ & $6.5 \times 10^{-4}$ & & & & & & & $1.606 \pm 0.35$ \\
\hline E. mitis & $5.74 \pm 1.89$ & & & & & & & $12.21 \pm 1.46$ & $29.38 \pm 2.58$ & $37.48 \pm 3.53$ & $38.83 \pm 4.98$ & & & $38.83 \pm 4.98$ \\
\hline & & & & & & & & & & & & & & \\
\hline daigremontian & $a 3.14 \pm 2.85$ & & & & & & & & $10.07 \pm 2.91$ & $11.58 \pm 1.56$ & $11.76 \pm 4.72$ & $11.76 \pm 4.72$ & & $11.76 \pm 4.72$ \\
\hline K. pinnata & $10.8 \pm 3.8$ & & & & & & & & $28.2 \pm 7.7$ & $30.9 \pm 7.1$ & $45.0 \pm 6.1$ & $55.8 \pm 3.6$ & $65.5 \pm 9.4$ & $65.5 \pm 9.4$ \\
\hline
\end{tabular}




\section{CUADRO 4}

Polinizadores y visitantes de cinco especies ornitófilas de un fragmento de bosque caducifolio secundario en Venezuela

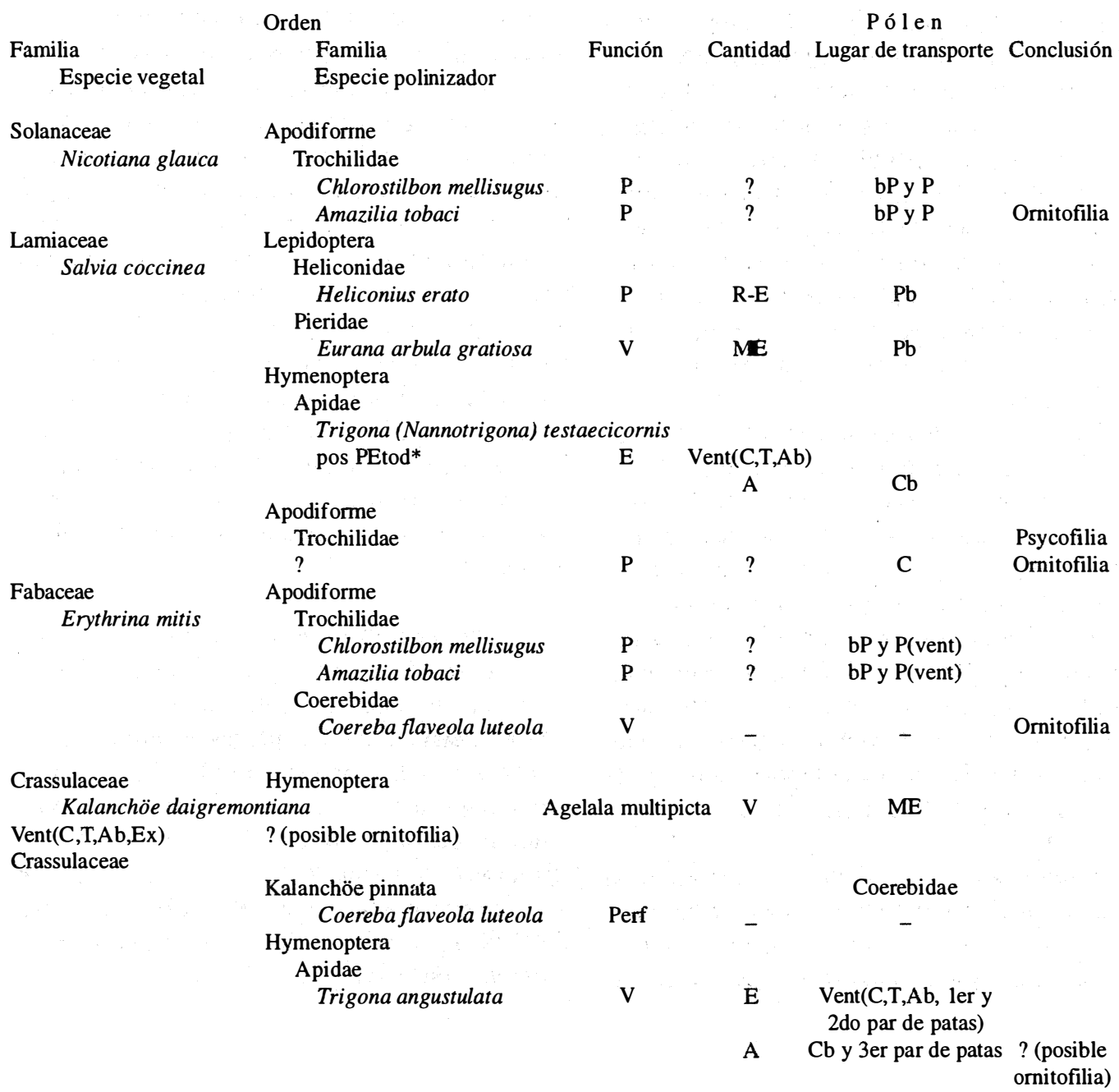

* Posible polinización etodinámica.

P, polinizador; V, visitante; Per, perforador; R, regular; E, escaso; ME, muy escaso; A, abundante; bP, base pico; P, pico; $\mathrm{Pb}$, proboscis; $\mathrm{C}$, cabeza; $\mathrm{T}$, torax; $\mathrm{Ab}$, abdomen; Ex, extremidades; Vent, ventral; $\mathrm{Cb}$, corbículas.

por $C$. flaveola luteola, con una tendencia a visitar esta especie constantemente durante el día, bajando un poco su actividad en horas del mediodía (entre las 12:00 y las 14:00). El polen de $K$. pinnata es colectado por Trigona angustulata (Apidae).

Sistema de apareamiento: Todas las especies fueron hermafroditas, dos de las cuales fueron adicógamas ( $N$. glauca y $S$. coccinea). El resto de las especies fueron protándras incompletas. Ninguna de las especies estudiadas resultó ser agamospérmica. Sólo $K$. daigremontiana resultó ser hercógama.

La comparación de los resultados de producción de frutos y semillas luego de pruebas controladas de fertilización cruzada y de autofertilización mostraron que $K$. daigremontiana 
y $K$. pinnata son autoincompatibles,xenógamas obligadas, y el resto resultaron ser autocompatibles, (Cuadro 5). En especies autoincompatibles, la producción de frutos y semillas en ambas pruebas fue significativamente diferente luego de realizar una prueba de G. La comparación de los resultados de producción de frutos y semillas para las pruebas controladas de autofertilización y polinización automática para las especies autocompatibles se muestra en la Cuadro 6. Todas resultaron ser no-autógamas. $K$. daigremontiana y $K$. pinnata presentaron procesos de autofertilización mecánica al término de su período de longevidad floral.

La relación polen/óvulo de las cinco especies estudiadas se muestra en el Cuadro 7. Esta relación abarcó desde 201,05 hasta 253,94 en especies xenógamas, y desde 166,23 hasta 6.051,42 para especies parcialmente autocompatibles. $K$. daigremontiana y $K$. pinnata también presentaron un mecanismo de reproducción asexual. Este consistió en la fragmentación de propágulos ubicados en el margen de las hojas. La caída de dichos propágulos genera la formación de una nueva planta a través de procesos no tratados en este estudio.

Biomasa: La asignación de biomasa total fue alta con respecto a otros sistemas de polinización, y no estuvo relacionada con el hábito de cada especie (Cuadro 8). Las relaciones biomasa reproductiva/biomasa vegetativa (BR/BV) y biomasa reproductiva/biomasa de atracción (BR/BA) fueron siempre menores a la unidad, lo cual indica que dentro de la flor, la asignación de recursos para las funciones vegetativas y de atracción siempre fueron mayores que la asignación para las funciones reproductivas (con la excepción de $K$. pinnata, en la cual la asignación de biomasa es igual tanto para la función reproductiva como para la de atracción). La relación biomasa androceo/biomasa gineceo (BAnd/Bgin) resultó ser mayor que 1 para $N$. glauca, $S$. coccinea y $E$. mitis, lo cual muestra que la asignación de biomasa dirigida hacia las estructuras reproductivas femeninas de estas especies es menor que la asignación de las estructuras masculinas. Caso contrario ocurre para $K$. daigremontiana y $K$. pinnata, en donde la relación resultó ser menor que 1.

Eficiencia natural: En general, S. coccinea y $N$. glauca son especies que presentaron bajos niveles de aborto global, sugiriendo una alta eficiencia en los procesos reproductivos (Cuadro 9). Las plantas introducidas presentaron altos niveles de aborto. Destaca el alto aborto de flores-frutos de E. mitis (90.82\%). También destacan el alto aborto de óvulos en E. mitis $(78.43 \%)$ y $K$. daigremontiana (71.60\%), y el alto aborto de semillas en $K$. pinnata (86.50\%).

CUADRO 5

Comparaciones entre autofertilizaciones y fertilizaciones cruzadas controladas de cinco especies de un fragmento de bosque caducifolio secundario en Venezuela

\begin{tabular}{lccccccccccc} 
Especie & \multicolumn{2}{c}{ Flores } & \multicolumn{2}{c}{ Frutos } & \multicolumn{2}{c}{ \% Frutos } & \multicolumn{2}{c}{ \% Semillas } & \multicolumn{2}{c}{ Test estadístico } & Conclusión \\
& & AF & FC & AF & FC & AF & FC & AF & FC & G fl-fr & G ov-se \\
N. glauca & 16 & 20 & 3 & 14 & 18.75 & 70,00 & 7.72 & 43.17 & 9.30 & 7.953 .36 & PAC \\
S. coccinea & 68 & 93 & 43 & 66 & 63.24 & 70,97 & 43.12 & 45.27 & 1.07 & 0.83 & AC \\
E. mitis & 36 & 23 & 3 & 13 & 8.33 & 56,52 & 2.65 & 12.44 & 0.40 & 36.42 & PAC \\
K. daigremontiana & 18 & 22 & 18 & 22 & 100 & 100 & 15.88 & 19.34 & 3.11 & 46.20 & AI \\
K. pinnata & 15 & 15 & 15 & 15 & 100 & 100 & 55.58 & 69.47 & 0 & 1.349 .52 & AI
\end{tabular}




\section{CUADRO 6}

Comparaciones entre autofertilizaciones y polinizaciones automáticas controladas de tres especies autocompatibles de un fragmento de bosque caducifolio secundario en Venezuela

\begin{tabular}{|c|c|c|c|c|c|c|c|c|c|c|c|}
\hline \multirow[t]{2}{*}{ Especie } & \multicolumn{2}{|c|}{ Flores } & \multicolumn{2}{|c|}{ Frutos } & \multicolumn{2}{|c|}{ \% Frutos } & \multicolumn{2}{|c|}{$\%$ Semillas } & \multicolumn{2}{|c|}{ Test estadístico } & \multirow[t]{2}{*}{ Conclusión } \\
\hline & $\mathrm{AF}$ & PA & $\mathrm{AF}$ & PA & $\mathrm{AF}$ & PA & $\mathrm{AF}$ & PA & $\mathrm{G} \mathrm{fl}-\mathrm{fr}$ & G ov-se & \\
\hline S. coccinea & 68 & 98 & 43 & 53 & 63.24 & 54.08 & 43.12 & 37.85 & 0.50 & 2.904 .58 & NAU \\
\hline$K$. daigremontiana & 18 & 31 & 18 & 31 & 100 & 100 & 15.88 & 12.28 & 1.04 & 14.35 & NAU \\
\hline$K$. pinnata & 15 & 15 & 15 & 15 & 100 & 100 & 55.58 & 44.06 & 0.17 & 0.97 & NAU \\
\hline
\end{tabular}

AF, autofertilización; PA, polinización automática; NAU, no- autógama,

\section{CUADRO 7}

Relación de los parámetros reproductivos masculinos y femeninos de cinco especies de un fragmento de bosque caducifolio secundario en Venezuela

\begin{tabular}{|c|c|c|c|c|c|c|c|}
\hline Especie & tambres/flor & $\begin{array}{c}\text { \# Granos de } \\
\text { polen/flor }\end{array}$ & $\begin{array}{c}\text { Area polen } \\
\left(\mathrm{mm}^{2}\right)\end{array}$ & Tipo estigma & $\begin{array}{l}\text { Area estigmática } \\
\qquad\left(\mathrm{mm}^{2}\right)\end{array}$ & $\mathrm{P} / \mathrm{O}$ & $\begin{array}{c}\text { Relación área } \\
\text { estig/Area } \\
\text { polen }\end{array}$ \\
\hline N. glauca & 5 & $214.583 .33 \pm 39.024 .56$ & $2.91 \times 10^{-3}$ & lobulado & $2.807 \pm 0.32$ & 166.23 & 964.60 \\
\hline S. coccinea & 2 & $5.360 .00 \pm 1.334 .059$ & $5.243 \times 10^{-3}$ & bífido desigual & $1.985 \pm 0.41$ & 1.343 .36 & 378.60 \\
\hline E. mitis & $9.98 \pm 0.14$ & $101.542 .86 \pm 20.622 .441$ & $4.25 \times 10^{-3}$ & clavado & $1.112 \pm 0.254$ & 6.051 .42 & 261.65 \\
\hline K. daigremontiano & 8 & $143.835 .2 \pm 21.070 .607$ & $2.64 \times 10^{-3}$ & capitado & $1.424 \pm 0.321$ & 253.94 & 539.39 \\
\hline$K$. pinnata & 8 & $369.233 .34 \pm 56.437 .184$ & $2.393 \times 10^{-3}$ & capitado & $1.47 \pm 0.303$ & 201.05 & 614.29 \\
\hline
\end{tabular}

\section{DISCUSIÓN}

Biología de polinización y biología floral: La floración de $N$. glauca promueve la presencia continua de recursos a los colibríes polinizadores durante todo el año. Entre los meses de diciembre y abril, $N$. glauca comparte dichos polinizadores con E. mitis. Esto sugiere que existe cierto grado de solapamiento por el polinizador. Este solapamiento se ve parcialmente disminuido por la deposición diferencial del polen sobre los polinizadores: E. mitis distribuye el polen sobre los polinizadores a lo largo de la zona ventral del pico y su base; y $N$. glauca lo hace a lo largo de todo el pico y su base. Dicha segregación espacial permite un menor solapamiento de las cargas de polen, y por ende, una menor interferencia entre los procesos de ambas especies (Campbell et al. 1994). Cabe destacar que especies de la misma sección taxonómica de E. mitis (sección Erythrina) son polinizadas naturalmente por colibríes (Neill 1987), lo cual sugiere que C. me- llisugus y A. tobaci son polinizadores sustitutos de sus polinizadores naturales. Por otra parte, la hora de antesis de las flores es particularmente importante en especies ornitófilas, ya que contribuye a la especificidad del sistema (Seres \& Ramírez 1995). Esta ocurrió mas temprano en especies cuya longevidad era pequeña. Probablemente, una antesis mas temprana contribuye a resolver parcialmente el solapamiento

Los colibríes resultaron ser polinizadores con mayor grado de especificidad, probablemente debido a que la exerción de los estambres de $S$. coccinea impide un contacto efectivo en el caso de agentes visitantes como lepidópteros e hymenópteros. Sin embargo, $H$. erato logra tener cierto contacto con las estructuras reproductivas de la flor de $S$. coccinea. En consecuencia, en la proboscis del animal se encuentra cierta cantidad de polen, aunque esta es baja. El comportamiento de T. testaecicornis en las flores de $S$. coccinea podría estar favoreciendo un mecanismo de polinización etodinámico en el cual el 
Asignación de biomasas a las estructuras reproductivas de cinco especies

de un fragmento de bosque caducifolio secundario en Venezuela

\begin{tabular}{|c|c|c|c|c|c|c|c|c|c|c|}
\hline \multirow[b]{2}{*}{ Especie } & \multirow[b]{2}{*}{$\mathrm{Cal}(\%)$} & \multirow[b]{2}{*}{ Cor $(\%)$} & \multirow[b]{2}{*}{ Fil (\%) } & \multirow[b]{2}{*}{ Ant (\%) } & \multirow[b]{2}{*}{$\operatorname{Gin}(\%)$} & \multicolumn{5}{|c|}{ Androceo (\%) } \\
\hline & & & & & & $\begin{array}{l}\text { Biomasa total } \\
(\mathrm{mg})\end{array}$ & Fruto $(\mathrm{g})$ & Rel And/C & $\mathrm{BR} / \mathrm{BV}$ & $\mathrm{BR} / \mathrm{B}$ \\
\hline glauca & $5.76 \pm 1.26(24.66)$ & $10.87 \pm 1.81(46.55)$ & $21(6.21)$ & $2.39 \pm 0.21$ & $2.88 \pm 0.52(12.33)$ & 23.35 & $0.12723 \pm 0.17552$ & 1.33 & 0.40 & \\
\hline coccinea & $0.580 \pm 0.135(22.90)$ & $1.1605 \pm 0.142(45.84)$ & $0.49 \pm 0$ & $06(19.35)$ & $0.302 \pm 0.133(11.92)$ & ) $2.5325 \quad 4$ & $4.2585 \times 10^{-3} \pm 0.716 \times 10^{-3}$ & 1.62 & 0.46 & \\
\hline mitis & $27.626 \pm 2.2(32.95)$ & $38.4 \pm 3.323(45.80)$ & $9.74 \pm 1.34(11.62)$ & $4.077 \pm 0.396(4.86)$ & $3.99 \pm 1.06(4.76)$ & 83.833 & $1.357 \pm 0.938$ & 3.46 & 0.27 & \\
\hline daigremontic & ana4.05 $\pm 1.53(16.54)$ & $10.99 \pm 1.76(44.89)$ & $1,84 \pm 0,33(7,52)$ & $2.54 \pm 0.23(10.38)$ & $5.06 \pm 0.65(20.67)$ & 24.486 & $6,945 \times 10^{-3} \pm 1.703 \times 10^{-3}$ & 0.86 & 0.63 . & \\
\hline pinnata & $28.69 \pm 2.061(30.22)$ & $32.53 \pm 4.56(34.27)$ & $6.64 \pm 2.62(6.99)$ & $5.33 \pm 0.401(5.61)$ & $21.74 \pm 2.124(22.90)$ & 94.93 & $0.03603 \pm 7.543 \times 10^{-3}$ & 0.55 & 0.55 & \\
\hline
\end{tabular}

Cal. cáliz; Cor. corola; Fil, filamentos; Ant, anteras; And,androceo; Gin, gineceo; BR, biomasa reproductiva; BV, biomasa vegetativa; BA, biomasa de atracción.

\section{CUADRO 9}

Eficiencias reproductivas de cinco especies de un fragmento de bosque caducifolio secundario en Venezuela

\section{Especie}

S. coccinea

E. mitis

K. daigremontiana

$K$. pinnata

\section{\#Flor/inflorescencia}

semillas

$24.30 \pm 20.58(\mathrm{n}=201)$

$27.54 \pm 10.56(\mathrm{n}=100)$

$39.53 \pm 20.51(n=148)$

$98.616 \pm 8.28(\mathrm{n}=101)$

$76.62 \pm 32.90(n=100)$
\#Frutos/ infrutescencia

$20.05 \pm 21.1(\mathrm{n}=231)$

$2.97 \pm 7.796(n=107)$

$3.63 \pm 3.17(n=165)$

$97.00 \pm 77.45(n=100)$

$66.53 \pm 53.98(n=100)$

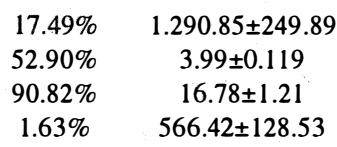

$566.42 \pm 128.53$

$1836.51 \pm 377.26$
\# Semillas/fruto

$\begin{array}{ccc}110.874 \pm 106.561 & 9.00 \% & 9.44 \% \\ 0 & 19.55 \% & 0 \% \\ 0.41 \pm 0.82 & 78.43 \% & 11.33 \% \\ 92.732 \pm 120.316 & 71.60 \% & 57.66 \% \\ 1.339 .84 \pm 531.53 & 15.66 \% & 86.50 \%\end{array}$

$3.21 \pm 0.799$
$3.21 \pm 1.59$
$159.3299 \pm 141.996$
$1548.9 \pm 546.36$

$1.339 .84 \pm 531.53$ 
polinizador se acerca voluntariamente a las estructuras reproductivas de la flor (Faegri \& van der Pijl 1979), aún cuando el polinizador no esté adaptado para el síndrome de polinización presente en la especie.

Debido al hecho de que $K$. pinnata y $K$. daigremontiana no reciben ninguna visita de colibrí, su presencia en esta comunidad no afecta los procesos de polinización de las especies nativas. Ambas especies exóticas presentaron visitas florales esporádicas, probablemente como consecuencia de que no son reconocidas por los polinizadores nativos. Es notable que ambas especies presentaron sus flores orientadas verticalmente hacia abajo. Esta disposición podría contribuir a reducir su conspicuidad y, consecuentemente, a disminuir su atractivo hacia los colibríes nativos. Dado lo fortuito de las visitas, la reproducción asexual presente en estas especies podría ser muy importante para el éxito reproductivo de las mismas en esta comunidad.

Debido a que las aves y las mariposas diurnas requieren atrayentes visuales (Faegri \& van der Pijl 1979), la biomasa total asignada a flores de especies ornitófilas y psicófilas es grande. Las especies que presentan mayores costos florales son $K$. pinnata y $K$. daigremontiana. Cabe destacar que estas especies también presentan las longevidades más prolongadas. Esto implica un alto costo de mantenimiento de la flor, lo cual sumado a las altas asignaciones de biomasa total presentes en estas especies, hacen de su costo floral uno aún más alto.

La producción de néctar en las especies estudiadas es alta en comparación con la de especies polinizadas por otro tipo de animales referidas por Stiles y Freeman (1993). Esto concuerda con las observaciones de Percival (1965), quién señala una alta producción de néctar como característica principal para la polinización por colibríes. Lo anterior sugiere que $S$. coccinea es una planta que, por su producción de néctar, presenta poco atractivo para los colibríes. Esto puede explicar la menor tasa de visitas. Por otro lado, $S$. coccinea fue la única especie que presentó valores de néctar acumulado menores que el total al final de su período de longevidad floral. Esto puede ser debido a la evaporación del néctar producto de la temperatura y/o de la reabsorción de néctar por parte de la planta. Cruden et al. (1983) señalan que la producción de néctar se detiene cuando se alcanza el máximo, y la reabsorción de éste comienza cuando los polinizadores están inactivos. Todo esto, coincide con el hecho de que la disminución del volumen de néctar producido comienza en horas del mediodía, en las cuales se alcanzan mayores temperaturas, $y$ los polinizadores se encuentran menos activos. Por lo tanto, es posible que ocurra una combinación de reabsorción y evaporación.

Sistema de apareamiento: La fertilización cruzada se ve favorecida a través de mecanismos de autoincompatibilidad genética (Gibbs 1990). Dichos mecanismos sólo están presentes en las dos especies de Kalanchöe, las cuales dirigen gran cantidad de sus recursos a la atracción de polinizadores (alta biomasa reproductiva, alta longevidad y alta producción de néctar), e indica que estas plantas presentan un alto costo reproductivo. Por otro lado, la dicogamia incompleta presente en $E$. mitis, $K$. daigremontiana y $K$. pinnata tiene particular importancia ya que contribuye a la reducción de la interferencia polen-estigma (LLoyd \& Webb 1986; Bertin \& Newman 1993). El proceso de autofertilización mecánica en ambas especies de Kalanchöe ocurre al final del período de longevidad floral lo cual promueve el contacto entre las partes reproductivas de la flor (LLoyd \& Webb 1986). Este proceso es de gran importancia en ausencia de polinizadores, y en especies autoincompatibles que producen alguna proporción de semillas por autofertilización.

Destaca el hecho que la relación P/O para $K$. daigremontiana, $K$. pinnata y $N$. glauca no concuerdan con los valores reportados por Cruden (1977) para especies xenógamas y xenógamas facultativas respectivamente. Estas tres especies producen un número elevado de óvulos por flor, lo cual probablemente afecta la relación $\mathrm{P} / \mathrm{O}$, independientemente de su sistema de apareamiento Dicha relación si concuerda con 
los valores que Cruden (1977) informa para especies parcialmente xenógamas, para el caso de $E$. mitis y $S$. coccinea. Cruden \& Jenzen (1979) exponen que bajos valores de P/O reflejan una afluencia suficiente de polen al estigma, lo cual parece estar relacionado con un transporte de polen muy eficiente. Todo esto sugiere que los polinizadores de $N$. glauca son animales altamente eficientes en el transporte y deposición de sus cargas de polen.

Biomasa: En general, la asignación de biomasa para la función reproductiva es menor en relación a las funciones de protección y de atracción, coincidiendo con los resultados de Antos y Allen (1994), los cuales obtuvieron que la biomasa de atracción constituía entre $50 \%$ y $63 \%$ de la biomasa reproductiva total de Oemleria cerasiformis. Probablemente, la atracción de los polinizadores constituye una presión selectiva mayor (Antos \& Allen 1994). Los resultados de las asignaciones de biomasa a las estructuras reproductivas y la relación BAnd/BGin muestran que existe una mayor asignación al androceo para $N$. glauca, S. coccinea y E. mittis. Esto coincide con los valores informados por Lovett y Cavers (1982), los cuales postulan, que existe una alta asignación de biomasa para las funciones masculinas en plantas xenógamas y xenógamas facultativas, y una alta asignación de biomasa para las funciones femeninas en plantas autógamas. Lovett y Cavers (1982) exponen que las plantas xenógamas y xenógamas facultativas presentan altos niveles de pérdida de polen producto del transporte por parte del polinizador, lo cual está asociado a una mayor asignación de recursos a la producción de granos de polen, incrementando la asignación total de biomasa a la función masculina.

Para el caso de las dos especies de Kalanchöe, las bajas relaciones de $\mathrm{P} / \mathrm{O}$ y las relaciones BAnd/BGin menores que uno en estas especies indican una coincidencia en el hecho de que estas especies presentan mayores asignaciones de recursos a la función femenina, lo cual contrasta con su sistema de apareamiento, difiriendo con los postulados de Lovett y Cavers
(1982). Estas dos especies también presentan una alta asignación de biomasa hacia las estructuras de atracción de polinizadores, lo cual podría estar creando una fuga de recursos. Por otro lado, es probable que el o los polinizadores naturales de estas especies presenten altísimos niveles de especificidad, reduciendo así la pérdida de polen por transporte o ingesta.

Eficiencia natural: La baja producción de semillas en $E$. mitis puede estar determinada porque sus polinizadores naturales no se encuentran en esta comunidad. Ahora bién, ¿porqué $E$. mitis presenta una eficiencia tan baja si presenta una alta tasa de visitas de sus polinizadores? El comportamiento de $C$. mellisugus y $A$. tobaci sobre $E$. mitis es tal que la cantidad de visitas legítimas que realizan es baja, lo cual da como resultado, una afluencia de polen coespecífico baja, y por ende, bajos niveles de fertilización cruzada; además de que solo hay tres individuos en la comunidad, lo cual dificulta aún mas el intercambio de polen. Si recordamos que $E$. mitis es una especie introducida con alta asignación de recursos a la biomasa total, longevidad floral prolongada y con una alta inversión de recursos para la producción de néctar, entonces probablemente sus bajos niveles de eficiencia reproductiva también podrían estar parcialmente asociados a la interferencia con $N$. glauca, ya que $C$. mellisugus y A. tobaci son polinizadores de ambas especies.

Los mayores porcentajes de aborto de óvulos y semillas se observaron en $K$. daigremontiana y $K$. pinnata. Esto probablemente pueda estar asociado a la ausencia de polinizadores $\mathrm{y}$, por consiguiente, a tasas mínimas de entrecruzamiento. En contraste, $S$. coccinea y $N$. glauca presentan una mayor eficiencia reproductiva. Ambas son especies nativas, lo cual muestra que las plantas nativas de una comunidad, en presencia de sus polinizadores naturales, son más eficientes que especies introducidas, a pesar de la perturbación del ambiente. Además, ambas especies crecen en condiciones secundarias, lo cual sugiere una adaptación a su reproducción bajo condiciones de perturbación. 
Las especies introducidas no afectan las eficiencias reproductivas de las especies nativas. Tal es el caso de $N$. glauca y E. mitis, donde ambas especies vegetales comparten sus polinizadores, pero el efecto de $E$. mitis (especie introducida) no parece afectar notoriamente los procesos de polinización de $N$. glauca, ya que esta presenta una alta eficiencia reproductiva. Las eficiencias reproductivas de las especies introducidas están drásticamente afectadas por la ausencia de sus polinizadores naturales. Aquellas especies cuyos polinizadores naturales presenten polinizadores sustitutos, del mismo tipo, en la comunidad a la cual son introducidas (E. mitis), presentan eficiencias reproductivas mayores que aquellas especies que no presenten dichos polinizadores sustitutos $(K$. daigremontiana y $K$. pinnata).

\section{AGRADECIMIENTOS}

Agradecemos a Maris López, Ivan Jaimes y Silvia Pérez por compartir algunos de sus resultados con nosotros; a Elena Raymúndez, Omaira Hokche y Maris López, por su colaboración en el transcurso del trabajo; a Nereida Xena, Thirza Ruíz y $\mathbf{M}^{\mathrm{a}}$ Angélica Taisma, por la revisión crítica del manuscrito. Al proyecto CONICIT S1/96001695.

\section{RESUMEN}

La biología floral, biología de polinización y sistema de apareamiento fueron estudiados en dos especies nativas $\mathrm{y}$ tres especies introducidas presentes en un fragmento de bosque caducifolio secundario en Venezuela. Todas las especies estudiadas presentaron corolas tubulares, largas y estrechas; predominando los colores rojos. La antesis de todas las especies fue diurna, entre las 04:00 y las 11:00. La producción de néctar fue alta, excepto para Salvia coccinea (Lamiaceae) (nativa). Nicotiana glauca (Solanaceae) (nativa) y Erythrina mitis (Fabaceae) (introducida) compartían sus polinizadores Chlorostilbon mellisugus y Amazilia tobaci (Apodiformes, Throchilidae). S. coccinea recibió visitas legítimas de Heliconius erato (Lepidóptera). Kalanchöe daigremontiana y $K$. pinnata (Crassulaceae) (exóticas) no recibieron ninguna visita legítima. Todas las especies fueron hermafroditas. $K$. daigremontiana y $K$. pinnata resultaron xenógamas obligadas; mientras $S$. coccinea, $N$. glauca y E. mitis resultaron parcialmente autocompatibles no autógamas. Las cinco especies mostraron una alta asignación de biomasa total con respecto a otros sistemas de polinización. La asignación de biomasa reproductiva fue siempre menor a las asignaciones de biomasa de atracción y de biomasa vegetativa floral. La asignación de biomasa masculina fue mayor que la femenina para $N$. glauca, $S$. coccinea y $E$. mitis, y menor para $K$. daigremontiana y $K$. pinnata. Las especies introducidas no parecen afectar notoriamente las eficiencias reproductivas de las especies nativas. Las eficiencias reproductivas de las especies nativas fueron superiores a las de las especies introducidas. Las eficiencias reproductivas de las especies introducidas resultaron bajas, debido a que se encuentran afectadas por la ausencia de sus polinizadores naturales.

\section{REFERENCIAS}

Antos J. A. \& G. A. Allen. 1994. Biomass allocation among reproductive structures in the dioecious shrub Oemleria cerasiformis - a functional interpretation. J. Ecol. 82: 21-29.

Bertin R. I. \& C. M. Newman. 1993. Dichogamy in angiosperms. Bot. Rev. 59: 112-152.

Campbell D. R., N. M. Waser \& M. V. Price. 1994. Indirect selection of stigma position in Ipomopsis aggregata via genetically correlated trait. Evolut. 48: 55-68.

Cruden R. W. 1977. Pollen-ovule ratios: a conservative indicator of breeding systems in flowering plants. Evolut. 31: 34-46.

Cruden R. W. \& K. G. Jensen. 1979. Vicsin threads; pollination efficiency and low pollen-ovule ratios. Amer. J. Bot. 66: 875-879.

Cruden R. W., S. M. Hermann \& S. Peterson. 1983. Patterns of nectar production and plant-pollinator coevolution, p. 80-125 In B. Bentley \& T. Elias (eds) The biology of nectaries. Columbia University, Nueva York.

Ewel, J., A. Madriz \& A. Tosi. 1976. Zonas de vida de Venezuela.Memoria explicativa sobre el mapa ecológico. M.A.C.-FONAIAP, Caracas, Venezuela. $270 \mathrm{p}$.

Faegri K. \& L. Van der pijl. 1979. The principles of pollination ecology. Pergamon, Oxford. 244 p.

Feinsinger P., K. G. Murray, S. Kinsman \& W. H. Busby. 1986. Floral neighborhood and polination success in four hummingbird-pollinated cloud forest plant species. Ecology 67: 449-464. 
Feinsinger P., J. H. Beach, Y. B. Linhart, W. H. Busby \& K. G. Murray. 1987. Disturbance, pollinator predictability, and pollination success among Costa Rican cloud forest plants. Ecology 68: 1294-1305.

Feinsinger P. \& H. M. Tiebout III. 1991. Competition among plants sharing hummingbird pollinators: laboratory experiments on a mechanism. Ecology 72: 1946-1952.

Feinsinger P., H. M. Tiebout III \& B. E. Young. 1991. Do tropical bird-pollinated plants exhibit density-dependent interactions? Field experiments. Ecology 72: 1953-1963.

Gibbs P. 1990. Self-compatibility in flowering plants: a neotropical perspective. Rev. Brasil. Bot. 13: 125-136.

Küppers H. 1979. Atlas de colores. Blume. Barcelona. 105 p.

Linhart Y. B., P. Feinsinger, J. H. Beach, W. H. Busby, K. G. Murray, W. Zuchowski Pounds, S. Kinsman, C. A. Guindon \& M. Kooiman. 1987. Disturbance and predictability of flowering patterns in bird-pollinated cloud forest plants. Ecology 68: 1696- 1710.

Lloyd D.G. 1965. Evolution of self-compatibility and racial differentiation in Leavenworthia (Cruciferae). Contr. Gray Herb. Harvard 195: 3-133.

Lloyd D. G. \& C. J. Webb. 1986. The avoidance of interference between the presentation of pollen and stigmas in angiosperms. I.- Dichogamy. New Zeal. J. Bot. 24: 135-162.

Lovett Doust J. \& P. B. Cavers. 1982. Biomass allocation in hermaphrodite flowers. Can. J. Bot. 60: 2530-2534.

Neill D. A. 1987. Trapliners in the trees: hummingbird pollination of Erythrina sect. Erythrina (Leguminosae: Papilionoideae). Ann. Miss. Bot. Gard. 74: 27-41

Percival M.S. 1965. Floral Biology. Pergamon, Londres p. 101-119.

Phelps W. \& R. de Schauensee. 1979. Una guía de la aves de Venezuela. Gráficas Armitano, Caracas, Venezuela. 484 p.
Ramírez N. 1992. Especificidad de los sistemas de polinización en una comunidad arbustiva de la Guayana venezolana. Ecotrópicos 5:1-19.

Ramírez N. 1993. Reproductive biology in a tropical shrubland of Venezuelan Guyana. J. Vegetation Sci. 4: 5-12.

Ramírez N. \& A. Seres. 1994. Plant reproductive biology of herbaceous monocots in a Venezuelan tropical cloud forest. Plant. Syst. Evolut. 190: 129144.

Ruiz-Zapata T. \& M. T. K. Arroyo. 1978. Plant reproductive ecology of a secondary deciduous tropical forest in Venezuela. Biotropica 10: 221-230.

Seres A. \& N. Ramírez. 1995. Biología floral y polinización de algunas monocotiledoneas de un bosque nublado venezolano. Ann. Miss. Bot. Gard. 82: 6181 .

Sobrevila C. \& M. T. K. Arroyo. 1982. Breeding system in a montane tropical cloud forest in Venezuela. Plant Syst. Evolut. 140: 19-37.

Stiles F. G. 1978. Temporal organization of flowering among the hummingbird foodplant of a tropical wet forest. Biotropica 10: 194-210.

Stiles F. G. 1979. El ciclo anual de una comunidad coadaptada de colibríes y flores en el bosque tropical muy húmedo de Costa Rica. Rev. Biol. Trop. 27: 75-101.

Stiles F. G. \& E. Freeman. 1993. Patterns in floral nectar characteristics of some bird-visited plant species from Costa Rica. Biotropica 25: 191-205.

Tamayo F. 1943. Notas ecológicas venezolanas: Proceso de despoblación y reposición vegetal de las colinas de Caracas. Talleres de Artes Gráficas, Caracas, Venezuela. $23 \mathrm{p}$.

Webb C. J. \& D. G. Lloyd. 1986. The avoidance of interference between the presentation of pollen and stigmas in angiosperms. II Herkogamy. New Zeal. J. Bot. 24: 163-178. 